\title{
Chemotherapy in Patients with Hereditary Angioedema
}

\author{
CRISTINA MORELLI ${ }^{1}$, VINCENZO FORMICA ${ }^{1}$, STEFANIA PELLICORI ${ }^{2}$, ANTONELLO MENGHI $^{3}$, \\ MARIA DOMENICA GUARINO ${ }^{4}$, ROBERTO PERRICONE ${ }^{4}$ and MARIO ROSELLI ${ }^{1}$ \\ ${ }^{1}$ Internal Medicine Department, Tor Vergata University Hospital, Medical Oncology Unit, Rome, Italy; \\ ${ }^{2}$ Unit of Gastrointestinal Medical Oncology and Neuroendocrine Tumors, \\ European Institute of Oncology, Milan, Italy; \\ ${ }^{3}$ Medical Oncology Unit, INI Grottaferrata, Rome, Italy; \\ ${ }^{4}$ Rheumatology, Allergology and Clinical Immunology, Department of Systems Medicinei, \\ Tor Vergata University Hospital, Rome, Italy
}

\begin{abstract}
Background: Hereditary angioedema (HAE) is an autosomal dominant hereditary disorder characterized by episodic swelling of many body regions (especially throat and abdomen), potentially triggered by medication. No data are available for HAE in patients with cancer assigned to standard chemotherapy. The aim of our study was to identify circulating mediators potentially predictive of acute $H A E$ attacks during chemotherapy. Patient and Methods: Repeated blood testing (approximately every week) for complement system members (C3, C4, CH50, C1 inhibitor, C1-inhibitor functional $C(Q)$ ), $D$-dimers and for routine haematochemistry were performed in a 42-year-old male affected by type $2 \mathrm{HAE}$ during standard adjuvant oxaliplatin/fluorouracil-based chemotherapy administered for stage III radically resected rectal cancer. Pre-medication with 1,000 U Berinert inhibitor C1 was administered every week throughout treatment. MannWhitney U-test was used to determine statistical differences in measures between the first 30 days of therapy and beyond day 30 of therapy. Results: Pre-chemotherapy values of tested variables (day 0) were: C3: $101 \mathrm{mg} / \mathrm{dl}, \mathrm{C} 4: 5.71 \mathrm{mg} / \mathrm{dl}$, CH5O: 74\%, Cl inhibitor: $43.4 \mathrm{mg} / \mathrm{dl}$, Cl-inhibitor functional: $18 \%$, C1Q: $150 \mathrm{mg} / \mathrm{dl}$, and D-dimers: $113 \mathrm{~g} / \mathrm{ml}$. A significant change in circulating values was observed for C3, D-dimers and Cl-inhibitor functional. Four HAE attacks were observed, they started from the forth cycle of treatment and all were manageable. Changes in C3,D-dimers and C1-inhibitor functional preceded the attacks. Conclusion: The stress induced by chemotherapy such a standard oxaliplatin/
\end{abstract}

Correspondence to: Cristina Morelli, MD, Medical Oncology Unit, Internal Medicine Department, Tor Vergata University Hospital, Viale Oxford 81, 00133 Rome, Italy. Tel: +39 0620908190, Fax: +390620903544, e-mail: cristina.morelli89@gmail.com

Key Words: Hereditary angioedema, chemotherapy, colon cancer, immunology. fluorouracil increases the risk of attacks in patients with HAE. However, circulating biomarkers such as D-dimers, $C 3$ and Cl-inhibitor functional may serve as early predictors of acute HAE crisis.

The complement system $(1,2)$ abbreviated as ' $\mathrm{C}$ ', is an essential part of the innate immune system, made up of numerous proteins, most of which are proteases that are themselves activated by proteolytic cleavage (Table I). They localize at the sites of infection and on the surface of altered cells and trigger a cascade of proteolytic steps ultimately resulting in potent inflammatory signals and lytic events. It is known that three distinct pathways are initially activated: the classical pathway, the so-called lectin pathway (initiated by mannose-binding lectin) (3), and the alternative pathway (triggered by the binding of spontaneous activated complement component 3 (C3) in plasma to a pathogen surface) (4). The classical pathway (5) is initiated by the binding of complement protein $1 \mathrm{q}(\mathrm{C} 1 \mathrm{q})$, which is part of the $\mathrm{C} 1$ complex, to an antigen-antibody complex at pathogen or host cell surfaces, allowing the activation of C1r. Activated C1r cleaves C1s, generating an active serine protease which in turn cleaves $\mathrm{C} 4$ and $\mathrm{C} 2$. These reactions result in the formation of $\mathrm{C} 3$ convertase (formed by $\mathrm{C} 4 \mathrm{aC} 2 \mathrm{~b}$ ), which cleaves $\mathrm{C} 3$ into $\mathrm{C} 3 \mathrm{a}$, an anaphylatoxin, and $\mathrm{C} 3 \mathrm{~b}$, which binds covalently to a pathogen or an abnormal cell surface and acts both as an opsonin and as a proteolytic enzyme for further signal cascade (Figure 1).

Because of its ability to be extremely damaging to host tissues, the complement system is physiologically regulated by complement control proteins, which, in the blood, are present at a higher concentration than the complement proteins themselves. In particular, the classical pathway is inhibited by human C1-inhibitor (C1-INH) (6), a serine protease inhibitor of several complement proteases $(\mathrm{C} 1 \mathrm{r}$, $\mathrm{C} 1 \mathrm{~s}$, mannose-associated serine protease 1 and 2 (MASP-1 and MASP-2). C1-INH also regulates coagulation events (by controlling plasma kallikrein and factor XIIa), vascular 
Table I. Key components of the complement system.

Function

\begin{tabular}{|c|c|c|}
\hline \multirow[t]{5}{*}{ Classical pathway } & $\mathrm{C} 1 \mathrm{q}$ & Binds $\mathrm{Fc}$ region of IgM and certain Ig subclasses \\
\hline & $\mathrm{C} 1 \mathrm{r}$ & Serine proteinase, cleaves $\mathrm{C} 1 \mathrm{~s}$ \\
\hline & $\mathrm{C} 1 \mathrm{~s}$ & Serine proteinase, cleaves $\mathrm{C} 4$ and $\mathrm{C} 2$ \\
\hline & $\mathrm{C} 4$ & Key component of the $\mathrm{C} 4 \mathrm{~b} 2 \mathrm{a}(\mathrm{C} 3$ convertase) complex \\
\hline & $\mathrm{C} 2$ & \\
\hline \multirow[t]{3}{*}{ Alternative pathway } & Factor D & Serine proteinase, activates factor B \\
\hline & Factor B & Serine proteinase, the $\mathrm{Bb}$ fragment has both $\mathrm{C} 3(\mathrm{C} 3 \mathrm{bBb}$ complex $)$ and $\mathrm{C} 5$ convertase activity \\
\hline & Properdin & Stabilizes the C3bBb complex \\
\hline Common pathway & $\mathrm{C} 3$ & Key component for amplification step generation of $\mathrm{C} 5$ convertase activity \\
\hline \multirow[t]{5}{*}{ Membrane attack complex } & $\mathrm{C} 5$ & First component (C5b) of the MAC \\
\hline & C6 & Binds $\mathrm{C} 5 \mathrm{~b}$ and $\mathrm{C} 7$ \\
\hline & $\mathrm{C} 7$ & Binds $\mathrm{C} 7$ and $\mathrm{C} 8$ \\
\hline & $\mathrm{C} 8$ & Binds $\mathrm{C} 8$ and $\mathrm{C} 9$ \\
\hline & $\mathrm{C} 9$ & Polymerizes to form large membrane pore \\
\hline
\end{tabular}

permeability and inflammatory responses. A decrease in $\mathrm{C} 1$ INH levels leads to the uncontrolled activation of the complement system [causing the decrease of complement levels (C4)] with subsequent increase in anaphylatoxins (C3, C5a), and chemotaxins (C3b) (7). Moreover, the decrease in $\mathrm{C} 1-\mathrm{INH}$ activity generates a significant rise in factor XIIa, the starting point of the intrinsic coagulation pathway and of the pre-kallikrein-kallikrein-bradykinin pathway $(8,9)$, which is involved in vasodilatation and vascular permeability and is recognized as being responsible for capillary leakage and subsequent interstitial oedema (10).

A reduced C1-INH activity underlies the molecular etiopathogenesis of hereditary angioedema (HAE), an autosomal-dominant hereditary disorder characterized by episodic attacks of tissue swelling potentially involving many body regions, caused by abnormality in the synthesis or secretion of C1-INH as a result of mutations in its gene on chromosome 11 . In one-fourth of cases, the mutation is de novo (11-13).

Its prevalence reported in the literature ranges from 1 in 10,000 to 1 in 150,000 (1). It has been estimated that patients can be debilitated by their symptoms for 20-100 days a year (14).

Three different forms of HAE have been recognized (15): Type 1 is the most frequent ( $85 \%$ of patients) and is associated with a low level of $\mathrm{C} 1$-INH, type 2 is characterized by normal or increased level of dysfunctional C1-INH (16), type 3 typically affects females and is caused by an activating mutation in the F12 gene, encoding the Hageman factor (FXII) with normal serum C1-INH level and C1 activity (17, 18). All forms of HAE show markedly reduced C1-INH activity and C4 levels, but normal C1Q levels.

The disease is characterized by episodic peripheral swelling of the hands and feet, or sometimes of the face, neck, tongue, larynx (primary cause of mortality because of its possible progression to asphyxiation) and genitalia due to the leakage of plasma into subcutaneous stroma and mucosa. The cutaneous oedema is non-pitting, non-urticarial, with illdefined margins, involving areas of the face, extremities and genitals. Patients may also present with episodic swelling of the bowel that is the cause of severe and spasmodic abdominal pain, vomiting and either constipation or diarrhoea. Acute attacks, occurring at a frequency that ranges from weekly to less than once yearly in most symptomatic untreated patients, may last 2 to 5 days. Individual patients need a personalized plan for the treatment of acute attacks (19). 17- $\alpha$-Alkylated attenuated androgens, such as danazol, stanozolol, oxandrolone, tibolone, and (unattenuated) methyl-testosterone $(20,21)$ are used to attenuate symptoms and prevent acute phases. The mechanism by which androgens increase the $\mathrm{C} 1-$ INH level remains unknown. Since C1-INH is produced primarily by cells in the liver, it has been hypothesized that androgens may induce hepatocytes to produce an increased amount of C1-INH (22). Alternatively, androgens may reduce the breakdown of C1-INH. Pharmacological prophylaxis can be used in high-risk situations for incident swelling by administering $\mathrm{C} 1$-INH concentrate, either plasma-derived or recombinant (e.g. Cinryze or Berinert $\mathrm{P}$ ), attenuated androgens or fresh frozen plasma. In overt acute attacks, replacement of C1-INH with plasma-derived products, bradykinin receptor antagonists (Icatibant), kallikrein inhibitor (Ecallantide), and recombinant C1-INH are used.

Even though attacks are usually spontaneous, clear triggering factors have been identified, such as local tissue trauma, surgery (23), emotional stress, menstruation and infection. Certain pharmacological agents are also able to unleash attacks, such as oral contraceptives (24) and angiotensin-converting-enzyme inhibitor inhibitors (25). 


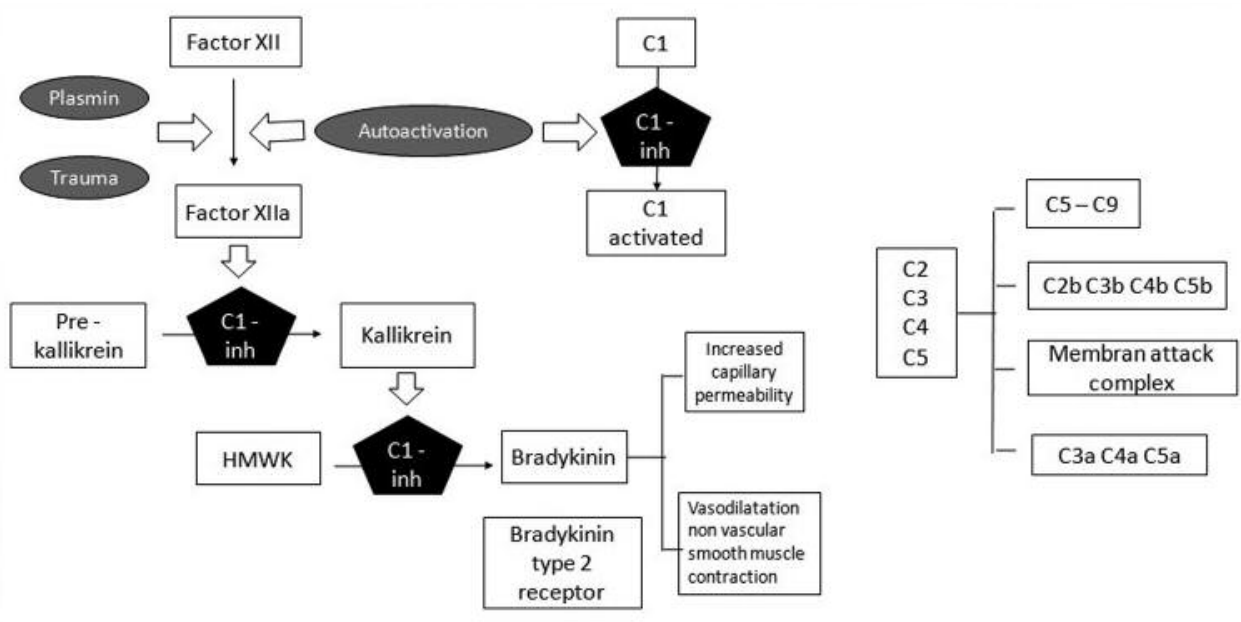

Stimulatory Inhibitory

Figure 1. Molecular etiopathogenesis of hereditary angioedema in the presence of contact system activators the zymogens factor XII (FXII); plasma pre-kallikrein $(P K)$ is converted into plasma kallikrein. These enzymatic forms active their zymogens; generating a positive feedback loop. PK proteolyzes high-molecular-weight kininogen $(H M W K)$ at two sites thus generating cleaved high-molecular-weight kininogen and the nonapeptide bradykinin in the kinin pathway. C1-inhibitor $(\mathrm{Cl}$-INH) controls the system by intervening at several locations.

Although anticancer treatments are highly toxic and likely to produce troublesome, stressful or even extremely dangerous reactions (such as mucositis, dermatitis or emesis), no data about their association with swelling attacks in patients with HAE are available.

In previous reports, anticancer hormone therapy in patients with breast cancer, such as tamoxifen, has been described as worsening swelling complications of HAE (26). However, few data are available on patients with cancer undergoing standard chemotherapy and radiotherapy for colorectal cancer.

The aim of this study was to observe the impact of a standard chemotherapy treatment on the risk of acute attacks in a patient with an established diagnosis of HAE undergoing adjuvant therapy for radically resected rectal cancer.

\section{Patient and Methods}

A 42-year-old employed male, current smoker of 20 cigarettes per day for 10 years had a previously established diagnosis of type 2 HAE for congenital deficiency of C1-INH. He had experienced the first attack when he was 12 years old, characterized by abdominal cramping, vomiting and diarrhoea and laryngeal enema with dyspnoea, and had been followed-up since then by the Immunology Department and usually treated with Berinert $\mathrm{C} 1$ esterase inhibitor (C1-INH) or plasma in case of acute attack. Because of bloody stools and changing of his bowel habitus, he underwent a colonoscopy in March 2013 and was diagnosed with a rectal adenocarcinoma, $1 \mathrm{~cm}$ from the anal verge, staged by endorectal ultrasound and a chest/abdomen/pelvis computed tomographic scan as cT3 $\mathrm{cN} 1$. According to the multidisciplinary team decision, the patient received neoadjuvant chemoradiation treatment, consisting of 5-fluorouracil (5-FU) for five cycles $\left(300 \mathrm{mg} / \mathrm{m}^{2} 5\right.$-FU i.v. day $1,20 \mathrm{mg} / \mathrm{m}^{2}$ leucovorin i.v. day 1 , weekly) and radiotherapy to the pelvis for a total dose of $4,500+540 \mathrm{cGy}$ in association with premedication with $1,000 \mathrm{U}$ Berinert inhibitor $\mathrm{C} 1$ in $100 \mathrm{ml}$ saline solution $i . v$. every week throughout treatment as prophylaxis against acute attacks. Only one attack of HAE was recorded after 2 weeks of chemoradiotherapy, characterized by abdominal pain with cramping and diarrhoea and oropharyngeal angioedema.

In July 2013, a repeat computed tomographic scan and endoscopic ultrasound confirmed tumour shrinkage (ycT2ycN0). At the end of July 2013, the patient underwent anterior rectocolic resection with ultra-low derivative ileostomy. The histological examination of the resected specimen confirmed the diagnosis of poorly differentiated adenocarcinoma with mucinous differentiation invading through the muscularis propria into pericolic fatty tissue, without lymph node involvement (ypT3N0 (0/2 nodes) Mx G3, wild-type Kirsten rat sarcoma 2 viral oncogene homolog $(K R A S)$, neuroblastoma rat sarcoma viral oncogene homolog (NRAS), v-Raf murine sarcoma viral oncogene homolog $\mathrm{B}(B R A F)$.

Two months after surgery, the patient was proposed adjuvant chemotherapy to complete 6 months of perioperative treatment. Considering the positive history of the patient for HAE and the frequent presentation of its attacks with gastrointestinal involvement, we discussed the case with the immunology team and decided to administer the OXAFAFU scheme $\left(85 \mathrm{mg} / \mathrm{m}^{2}\right.$ oxaliplatin i.v. day $1,850 \mathrm{mg} / \mathrm{m}^{2} 5$-FU i.v. day $2,250 \mathrm{mg} / \mathrm{m}^{2}$ folinic acid i.v. day 2) (27) for eight cycles with pre-medication of $1000 \mathrm{U}$ Berinert inhibitor $\mathrm{C} 1$ in $100 \mathrm{ml}$ saline solution i.v. every week throughout treatment. After signing informed consent, during adjuvant 
Table II. Values of tested variables throughout the whole chemotherapy period.

\begin{tabular}{|c|c|c|c|c|c|c|c|c|c|c|c|c|c|c|c|c|c|c|}
\hline $\begin{array}{l}\text { Day } \\
\text { of } \\
\text { CHT }\end{array}$ & $\begin{array}{l}\mathrm{CHT} \\
\text { cycle }\end{array}$ & $\begin{array}{c}\mathrm{C} 3 \\
(\mathrm{mg} / \mathrm{dl})\end{array}$ & $\begin{array}{c}\mathrm{C} 4 \\
(\mathrm{mg} / \mathrm{dl})\end{array}$ & $\begin{array}{c}\text { CH50 } \\
(\%)\end{array}$ & $\begin{array}{c}\mathrm{C} 1 \\
\mathrm{INH} \\
(\mathrm{mg} / \mathrm{dl})\end{array}$ & $\begin{array}{c}\text { C1 INH } \\
\text { FUNCT } \\
\quad(\%)\end{array}$ & $\begin{array}{c}\mathrm{C} 1 \mathrm{Q} \\
(\mathrm{mg} / \mathrm{l})\end{array}$ & $\begin{array}{l}\mathrm{Hb} \\
(\mathrm{g} / \mathrm{dl})\end{array}$ & $\begin{array}{c}\text { RBC } \\
\left(10^{6} / \mathrm{ml}\right)\end{array}$ & $\begin{array}{c}\text { WBC } \\
\left(10^{6} / \mathrm{ml}\right)\end{array}$ & $\begin{array}{l}\text { NEUT } \\
\left(10^{3} / \mathrm{ml}\right)\end{array}$ & $\begin{array}{l}\text { LYMPH } \\
\left(10^{3} / \mathrm{ml}\right)\end{array}$ & $\begin{array}{l}\text { EOSIN } \\
\left(10^{3} / \mathrm{ml}\right)\end{array}$ & $\begin{array}{c}\text { PLT } \\
\left(10^{3} / \mathrm{ml}\right)\end{array}$ & $\begin{array}{l}\text { DDIM } \\
(\mu \mathrm{g} / \mathrm{dl})\end{array}$ & $\begin{array}{c}\text { CEA } \\
(\mathrm{ng} / \mathrm{ml})\end{array}$ & $\begin{array}{l}\text { CA19.9 } \\
\text { (UI/ml) }\end{array}$ & $\begin{array}{c}\text { No. of } \\
\text { AE } \\
\text { attacks }\end{array}$ \\
\hline 0 & 1 & 101 & 5.71 & 74 & 43.4 & 18 & 150 & NA & NA & NA & NA & NA & NA & NA & NA & NA & NA & 0 \\
\hline 1 & 1 & 97.8 & 5.54 & 58 & 36.5 & 7 & 87 & NA & NA & NA & NA & NA & NA & NA & NA & NA & NA & 0 \\
\hline 2 & 1 & 68.9 & 3.53 & 14 & 24 & 0 & 61 & NA & NA & NA & NA & NA & NA & NA & NA & NA & NA & 0 \\
\hline 7 & 1 & 117 & 2.48 & 47.9 & 24 & 0 & 107.9 & 13.3 & 4.71 & 3.37 & 2.15 & 0.9 & 0.06 & 174 & 113 & 0.73 & 4.89 & 0 \\
\hline 13 & 1 & NA & NA & NA & NA & NA & NA & 12.8 & 4.53 & 5.68 & 3.58 & 1.38 & 0.18 & 199 & NA & $\mathrm{NA}$ & NA & 0 \\
\hline 15 & 2 & 119 & 2.17 & 5 & 24 & 0 & 96.7 & NA & NA & NA & NA & NA & NA & NA & NA & NA & NA & 0 \\
\hline 22 & 2 & 114 & 1.85 & 4 & 27.5 & 0 & 140 & 12.7 & 4.54 & 2.16 & 0.74 & 0.94 & 0.08 & 172 & 421 & NA & NA & 0 \\
\hline 28 & 3 & 118 & 2.47 & 4 & 35 & 37.7 & 153 & 12.3 & 4.47 & 4.81 & 3 & 1.07 & 0.11 & 150 & NA & 0.94 & $<1.2$ & 0 \\
\hline 30 & 3 & 115 & $<1.5$ & 4 & 30 & NA & NA & NA & NA & NA & NA & NA & NA & NA & NA & NA & NA & 0 \\
\hline 36 & 3 & 117 & 2.86 & 9 & 32.5 & 44 & NA & 12.7 & 4.58 & 2.04 & 0.85 & 0.72 & 0.05 & 112 & NA & 0.93 & 4.82 & 0 \\
\hline 38 & 3 & NA & NA & NA & NA & NA & NA & 14.9 & 5.08 & 6.03 & 2.53 & 2.19 & 0.16 & 122 & 645 & NA & NA & 0 \\
\hline 42 & 3 & 123 & 3.47 & 4 & 33.8 & 40 & 125 & 12.2 & 4.32 & 3.04 & 1.63 & 0.89 & 0.08 & NA & NA & NA & NA & 0 \\
\hline 49 & 4 & 117 & 4.31 & 37 & 35.1 & 36 & 122 & NA & NA & NA & NA & NA & NA & NA & NA & NA & NA & 1 \\
\hline 55 & 4 & 136 & 2.11 & 30 & 35.1 & 30 & 153 & 13.8 & 4.76 & 4.3 & 2.29 & 1.26 & 0.14 & 126 & 645 & 0.94 & 6.19 & 0 \\
\hline 59 & 4 & 133 & 1.77 & 5.3 & 32.5 & 40 & 144 & 12.5 & 4.23 & 5.32 & 3.27 & 1.4 & 0.12 & 82 & NA & NA & NA & 0 \\
\hline 63 & 5 & NA & NA & NA & NA & NA & NA & 12.5 & 4.2 & 4.57 & 2.95 & 1.03 & 0.08 & 108 & NA & NA & NA & 0 \\
\hline 64 & 5 & 117 & 5.27 & 4 & 33.8 & 25.9 & 138 & NA & NA & NA & NA & NA & NA & NA & NA & NA & NA & 0 \\
\hline 70 & 5 & 132 & 2.47 & 10 & 28.8 & NA & 147 & 13 & 4.37 & 1.83 & 0.5 & 0.69 & 0.14 & 123 & 779 & 1.12 & $<1.2$ & 0 \\
\hline 72 & 5 & NA & NA & NA & NA & NA & NA & NA & NA & NA & NA & 1.08 & 0.07 & 124 & NA & NA & NA & 1 \\
\hline 76 & 5 & 146 & 4.65 & 130.2 & 35.1 & 44.9 & 121.2 & 13.2 & 4.5 & 4.58 & 2.43 & 1.37 & 0.18 & 103 & NA & NA & NA & 0 \\
\hline 84 & 6 & NA & NA & NA & NA & NA & NA & 15.4 & 5.04 & 5.69 & 3.46 & 1.42 & 0.06 & 74 & NA & NA & NA & 4 \\
\hline 85 & 6 & NA & NA & NA & NA & NA & NA & 12.1 & 4.04 & 3.02 & 1.55 & 0.9 & 0.1 & 64 & 5701 & 1.28 & 0.29 & 4 \\
\hline 94 & 6 & NA & NA & NA & NA & NA & NA & 14.7 & 4.72 & 5.61 & 3 & 1.84 & 0.16 & 148 & NA & NA & NA & 0 \\
\hline 111 & 7 & NA & NA & NA & NA & NA & NA & 16.1 & 5.23 & 20.71 & 16.59 & 2.69 & 0.14 & 225 & 4781 & NA & NA & 0 \\
\hline 112 & 7 & NA & NA & NA & NA & NA & NA & 14.3 & 4.67 & 13.39 & 10.67 & 1.59 & 0.11 & 176 & 8114 & 1.27 & 9.6 & 0 \\
\hline 112 & 7 & NA & NA & NA & NA & NA & NA & 15.7 & 4.95 & 19.08 & 16.28 & 1.66 & 0.05 & 201 & NA & NA & NA & 0 \\
\hline
\end{tabular}

NA: Not available; CHT: chemotherapy; C1s: Complement component 1 subcomponent; C1r: Complement component 1r; C1q: complement protein 1q; C2-9: complement components 2-9; CH50: total complement activity; C1 INH: C1 inhibitor; C1 INH FUNCT: C1-inhibitor functional activities; Hb: hemoglobin; RBC: red blood cells; WBC: white blood cell count; NEUT: neutrophil count; LYMPH: lymphocyte count; EOSIN: eosinophil count; PLT: platelet count; DDIM: D-dimer; CEA: carcinoembryonic antigen; CA19.9: carbohydrate antigen 19.9; AE: angioedema.

chemotherapy routine blood tests including coagulation, D-dimers and blood count were prospectively performed together with HAEspecific tests (C3, C4, C1Q, C1-INH quantitative and functional tests) to identify putative predictors of acute attacks.

Potential predictors of acute attacks were expected to be those involved in inflammatory reactions and coagulative cascade activation. HAE-specific tests were also performed, which were: Plasmatic antigenic measurements of C1-INH (normal value $=15.40-35.10 \mathrm{mg} / \mathrm{dl}$ ) and $\mathrm{C} 1 \mathrm{q}$ (normal value $=50-250 \mathrm{mg} / \mathrm{l}$ ), which were performed by radial immunodiffusion [NOR-Partigen and (for C1q) LC-Partigen; Behring, Marburg, Germany] according to the manufacturer's instructions; plasmatic C3 (normal value $=90-180 \mathrm{mg} / \mathrm{dl}$ ) and $\mathrm{C} 4$ (normal value $=10$ $40 \mathrm{mg} / \mathrm{dl}$ ) were measured by chemiluminescence (Siemens Dimension Vista ${ }^{\circledR}$ System Healthineers Italy); CH50 activity of serum (normal value $=51-150 \%$ ) assessed by haemolytic assay (Diamedix EZ Complement Cells) and C1-INH functional activity (normal value $=70$ $130 \%$ ) assessed by a chromogenic assay (Berichrom ${ }^{\circledR}$ C1-Inhibitor; Siemens). Blood counts and D-dimers were routinely assessed before and during treatment.

Statistical significance in the changes of assessed variables during chemotherapy was analysed by comparing the difference between the values of the variables in the first part of chemotherapy (first month) versus the second part of chemotherapy, i.e. beyond the first month, using the Mann-Whitney's $U$-test $(p<0.05$ acceptable significance).

\section{Results}

The treatment was well tolerated for the first four cycles, with manageable haematological toxicity (neutropenia grade 2 and thrombocytopenia grade 3-4) and oxaliplatin-related grade 1 neuropathy. Starting from the fourth chemotherapy cycle, an increase in acute HAE attacks was observed. As the risk of attacks might have been increased by the stress associated with blood sampling, regular blood testing was interrupted at the end of the fifth cycle. Nonetheless, the number of HAE crises continued to increase to a maximum of four attacks in the period between the sixth and the seventh cycle, which was the reason for a $50 \%$ reduction in the dose of chemotherapy in the seventh cycle of treatment. Subsequently, in agreement with the immunology team, it was decided not to administer the last chemotherapy cycle because of the worsening risk of 
HAE attack. At the end of January 2014, the patient underwent a positron-emission tomography-computed tomographic scan which was negative for disease relapse. A regular oncological 3-to 6-month follow-up was then started. The patient is currently disease-free (last visit on October 2018). Regarding laboratory findings, pre-chemotherapy values of tested variables (day 0) were: C3: $101 \mathrm{mg} / \mathrm{dl}, \mathrm{C} 4$ : $5.71 \mathrm{mg} / \mathrm{dl}, \mathrm{CH} 50:$ 74\%, C1-INH: $43.4 \mathrm{mg} / \mathrm{dl}, \mathrm{C} 1-\mathrm{INH}$ functional. $18 \%, \mathrm{C} 1 \mathrm{Q}: 150 \mathrm{mg} / \mathrm{dl}$ and D-dimers: $113 \mu \mathrm{g} / \mathrm{ml}$. The starting of the angioedema attacks after the fourth cycle was associated with a progressive increase in D-dimers, C3, $\mathrm{C} 4$ and $\mathrm{CH} 50$ values as demonstrated by the laboratory tests, representing a progressive increase in the inflammatory state of the patient and a hyperactivation of the complement system. An increase of C3 level was observed during the period between the fifth and the sixth cycle, concurrently with attacks. An unexpected increase in C4, initially strong at the first cycle, then declined until the third cycle (double in the last two cycles), there was a similar trend for $\mathrm{CH} 50$ and $\mathrm{C} 1-$ INH. C1-INH functional activity increased by the third cycle, showing substantial differences in the measured values on the day of treatment and during the rest period $(80 \%$ between the two in the fifth cycle). C1q increased by more than $100 \%$ between the first and the third cycles, then tending to stability. D-dimers increased by $470 \%$ between the first and the third cycles (in concomitance with the first attack) and $780 \%$ between the third and the sixth one (four attacks) (Table II).

To assess the predictive role of the changes of investigated variables during chemotherapy, the mean values of each biomarker were calculated for the first 30 days of treatment ( 8 tests) and for the subsequent treatment period (beyond day 30, another 8 tests). According to the Mann-Whitney $U$-test, a significant change in values was observed for $\mathrm{C} 3$ $(p=0.009), \mathrm{D}$-dimers $(p=0.0008)$ and $\mathrm{C} 1$-INH functional $(p=0.01)$ (Figure 2).

The attacks having occurred in the second period of chemotherapy, these three variables with significant differences between the first and second parts of chemotherapy may be regarded as candidate predictors of acute HAE attack.

\section{Discussion}

To the best of our knowledge, this is the first report on the safety of adjuvant oxaliplatin/fluorouracil chemotherapy in a patient with hereditary angioedema. Literature reports only few cases of patients affected by hereditary angioedema and treated for a neoplasm.

Kasamatsu et al. in Japan reported on a 69-year-old man affected by HAE and lung cancer who developed acute attacks of angioedema during chemotherapy (28). Attacks were managed by self-administration of $\mathrm{C} 1$-INH and for this reason danazol was given during concurrent chemoradiotherapy

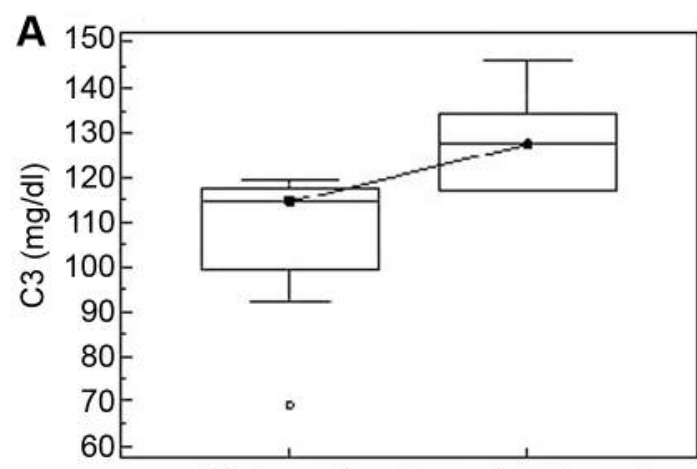

First month Beyond first month

B
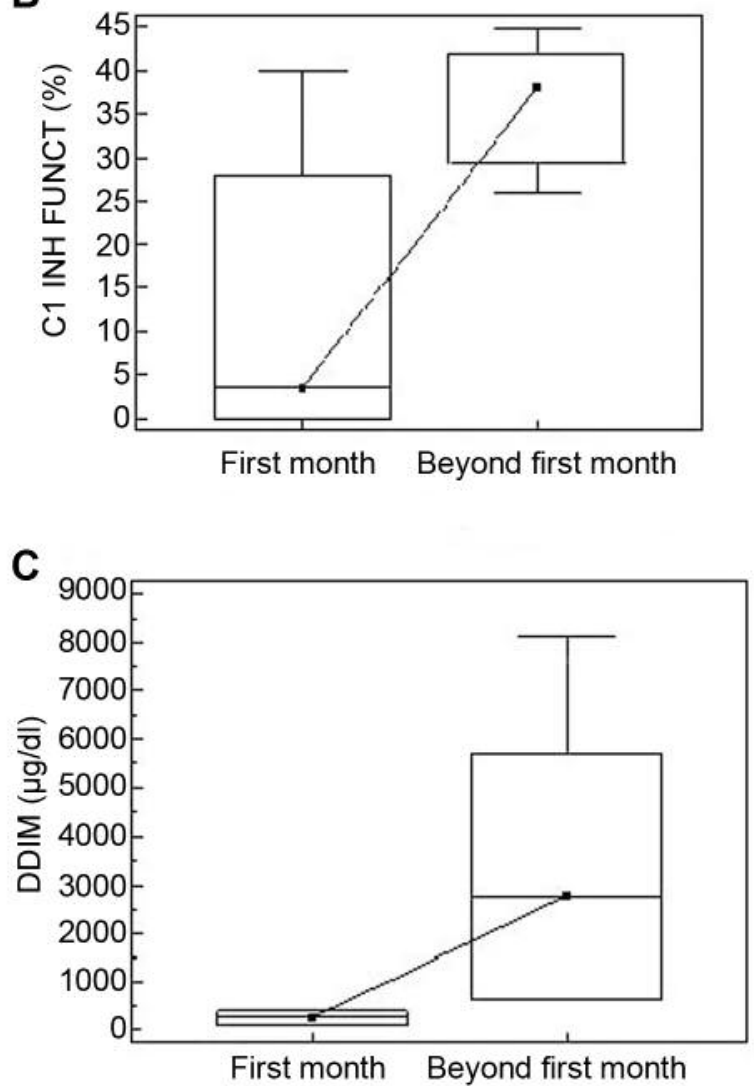

Figure 2. Comparison between mean values of complement component 3 (C3) (A); C1-inhibitor functional activities (C1-INH functional) (B) and D-dimers (DDIM) (C) calculated for the first 30 days of treatment and for the subsequent treatment period.

preventing further occurrence of angioedema attacks Christie et al. described the association between HAE and breast cancer in a family in which these diseases coexisted (29). Only one member of this family underwent surgery followed by adjuvant cyclophosphamide, methotrexate and 5-FU chemotherapy, she was given a course of radiation therapy in 
association with danazol without developing severe reactions or any angioedema attack. Rousset-Jablonski et al.] reported a case of HAE exacerbated by tamoxifen. They proposed that as it mimics oestrogen action in HAE, tamoxifen should be avoided in this special set of patients (26).

In the experience, our patient was affected by HAE and being treated for colon rectal cancer with administration of 5FU as single drug or in combination with oxaliplatin, the important stress induced by chemotherapy increased the risk of attacks, especially in the last part of the adjuvant treatment plan. The fact that we were able to deliver a satisfactory dose of adjuvant chemotherapy to this patient without major HAErelated complications is encouraging, and suggests that chemotherapy may be safely administered to patients with cancer with this rare inherited disease if regular pre-medication with $1000 \mathrm{U}$ of Berinert inhibitor $\mathrm{C} 1$ is applied in $100 \mathrm{ml}$ of physiological solution i.v. every week throughout treatment.

Nevertheless, the number of attacks at the end of the chemotherapy was significant, despite the prophylactic measures, confirming its potential role as trigger of HAE attacks.

The lack of data concerning the use of chemotherapeutic drugs in this particular set of patients generates many uncertainties in relation to unexpected interplay of these group of drugs with complement immunological pathways

The role of D-dimers has been previously evaluated in patients with HAE (30-32). Csuka et al. measured 11 parameters [factors XI, XII, and C1-INH activity; concentrations of D-dimers, prothrombin fragments 1 and 2, plasminogen, plasminogen activator inhibitor-1 (PAI1), thrombin-anti-thrombin III (TAT) complex, fibrinogen] and compared these markers in samples obtained from the same 39 patients during attack-free periods and during 62 edematous episodes. They demonstrated the increase of factor XII activity, as well as of the concentration of Ddimers, prothrombin fragments 1 and 2, and TAT during edematous episodes; and the PAI1 level, prothrombin time, and activated partial thromboplastin time significantly decreased during attacks, compared with symptom-free periods. The D-dimers level was significantly higher during multiple- $v s$. single-site attacks.

The statistically significant correlation we found between the increase of the complement activity markers and D-dimers and the concomitant increase of the number of acute attacks of HAE on the other, suggests a possible predictive role of these serum parameters in the clinical events connected to HAE, which warrants confirmation in further case reports.

As a conclusion, we believe that there is evidence that patients affected by HAE undergoing chemotherapy for an oncological disease need frequent and careful clinical monitoring. In our opinion, reducing the risk of acute HAE events in this specific set of patients is important during chemotherapy delivery because, firstly, this would increase the rate of full chemotherapy delivery and secondly, it would contribute to maintaining a good quality of life. We suggest two main research channels in this regard: The first is the possibility of finding serum markers that can predict acute HAE events at a useful time before their occurrence, and the second is to explore the feasibility of a prophylactic specific pre-medication that would prevent such events. The combination of these two courses would contribute to enabling physicians to predict and prevent acute attacks in patients with HAE undergoing chemotherapy.

\section{Funding}

There was no source of funding for this study.

\section{Conflicts of Interest}

The Authors declare that they have no conflict of interests in regard to this study.

\section{Ethical Approval}

All procedures performed in studies involving human participants were in accordance with the ethical standards of the institutional and/or national research committee and with the 1964 Helsinki declaration and its later amendments or comparable ethical standards.

\section{Informed Consent}

Informed consent was obtained from all individual participants included in the study.

\section{References}

1 Ricklin D, Hajishengallis $\mathrm{G}$, Yang $\mathrm{K}$ and Lambris JD: Complement: a key system for immune surveillance and homeostasis. Nature Immunol 11(9): 785-797, 2010.

2 Freeley S, Kemper C and Le Friec G: The "ins and outs" of complement-driven immune responses. Immunol Rev 274: 1632, 2016.

3 Troldborg A, Hansen A, Hansen SW, Jensenius JC, StengaardPedersen $\mathrm{K}$ and Thiel S: Lectin complement pathway proteins in healthy individuals. Clin Exp Immunol 188(1): 138-147, 2017.

4 Lachmann PJ: Looking back on the alternative complement pathway. Immunobiology 223(8-9): 519-523, 2018.

5 Kishore U, Thielens NM and Gaboriaud C: Editorial: State-ofthe-Art Research on $\mathrm{C} 1 \mathrm{q}$ and the Classical Complement Pathway. Front Immunol 7: 398, 2016.

6 Aulak KS, Donaldson VH, Coutinho M and Davis AE 3rd: C1inhibitor: structure/function and biologic role. Behring Inst Mitt (93): 204-13, 1993.

7 Klos A, Tenner AJ, Johswich KO, Ager RR, Reis ES and Kohl $\mathrm{J}$ : The role of the anaphylatoxins in health and disease. Mol Immunol 46: 2753-2766, 2009.

8 Nzeako UC, Frigas E and Tremaine WJ: Hereditary angioedema: a broad review for clinicians. Arch Intern Med 161(20): 24172429, 2001 
9 Madsen DE, Sidelmann JJ, Biltoft D, Gram J and Hansen S: C1inhibitor polymers activate the FXII-dependent kallikrein-kinin system: Implication for a role in hereditary angioedema. Biochim Biophys Acta 1850(6): 1336-1342, 2015.

10 Davis AE: 3rd. The pathophysiology of hereditary angioedema. Clin Immunol 114(1): 3-9, 2005.

11 Longhurst $\mathrm{H}$ and Cicardi M: Hereditary angio-oedema. Lancet 379(9814): 474-481, 2012.

12 Banerji A: Hereditary angioedema: classification, pathogenesis, and diagnosis Allergy Asthma Proc 32(6): 403-407, 2011.

13 Frank MM: 8. Hereditary angioedema. J Allergy Clin Immunol 121(2 Suppl): S398-401, 2008.

14 Cicardi $\mathrm{M}$ and Agostoni A: Hereditary angioedema. N Engl J Med 334: 1666-1667, 1996.

15 Cicardi M, Aberer W, Banerji A, Bas M, Bernstein JA, Bork K, Caballero T, Farkas H, Grumach A, Kaplan AP, Riedl MA, Triggiani $\mathrm{M}$, Zanichelli A and Zuraw B: HAWK under the patronage of EAACI (European Academy of Allergy and Clinical Immunology). Classification, diagnosis, and approach to treatment for angioedema: consensus report from the Hereditary Angioedema International Working Group. Allergy 69(5): 602-616, 2014.

16 Rosen FS, Charache P, Pensky and Donaldson V: Hereditary Angioneurotic Edema: Two Genetic Variants. Science 148: 956957, 1965

17 Bork K, Gul D and Dewald G: Hereditary angio-oedema with normal C1-INH in a family with affected women and men. Br J Dermatol 154: 542-545, 2006.

18 Björkqvist J, de Maat S, Lewandrowski U, Di Gennaro A, Oschatz C, Schönig K, Nöthen MM, Drouet C, Braley H, Nolte MW, Sickmann A, Panousis C, Maas C and Renné T: Defective glycosylation of coagulation factor XII underlies hereditary angioedema type III. J Clin Invest 125(8): 3132-3146, 2015.

19 Antoniu SA: Therapeutic approaches in hereditary angioedema. Clin Rev Allergy Immunol 41(1): 114-122, 2011.

20 Gelfand JA, Sherins RJ, Alling DW and Frank MM: Treatment of hereditary angio-oedema with danazol: reversal of clinical and biochemical abnormalities. N Engl J Med 295: 1444-1448, 1976.

21 Sheffer AL, Fearon DT and Austen KF: Clinical and biochemical effects of stanozolol therapy for hereditary angio-oedema. J Allergy Clin Immunol 68: 181-87, 1981.

22 Bowen T, Cicardi M, Farkas H, Bork K, Kreuz W, Zingale L, Varga L, Martinez-Saguer I, Aygören-Pürsün E, Binkley K, Zuraw B, Davis A, Hebert J, Ritchie B, Burnham J, Castaldo A, Menendez A, Nagy I, Harmat G, Bucher C, Lacuesta G, Issekutz A, Warrington R, Yang W, Dean J, Kanani A, Stark D, McCusker C, Wagner E, Rivard GE, Leith E, Tsai E, MacSween M, Lyanga J, Serushago B, Leznoff A, Waserman S and de Serres J: Canadian 2003 International Consensus Algorithm For the Diagnosis, Therapy, and Management of Hereditary Angioedema. J Allergy Clin Immunol 114: 629-637, 2004.
23 Aygören-Pürsün E, Martinez Saguer I, Kreuz W, Klingebiel T and Schwabe D: Risk of angioedema following invasive or surgical procedures in HAE type I and II - the natural history. Allergy 68(8): 1034-1039, 2013.

24 Giard C, Nicolie B, Drouet M, Lefebvre-Lacoeuille C, Le Sellin J, Bonneau JC, Maillard H, Rénier G, Cichon S, Ponard D, Drouet $\mathrm{C}$ and Martin L: Angio-oedema induced by oestrogen contraceptives is mediated by bradykinin and is frequently associated with urticaria. Dermatology 225(1): 62-69, 2012.

25 Baram M, Kommuri A, Sellers SA and Cohn JR: ACE inhibitorinduced angioedema. J Allergy Clin Immunol Pract 1(5): 442445, 2013.

26 Rousset-Jablonski C, Thalabard JC and Gompel A: Tamoxifen contraindicated in women with hereditary angioedema? Ann Oncol 20(7): 1281-1282, 2009.

27 Comella P, Massidda B, Filippelli G, Palmeri S, Natale D, Farris A, De Vita F, Buzzi F, Tafuto S, Maiorino L, Mancarella S, Leo S, Lorusso V, De Lucia L and Roselli M: Oxaliplatin plus high dose folinic acid and 5-fluoruracil I.V. bolus (OXAFAFU) versus irinotecan plus high dose folinic acid and 5-fluoruracil I.V. bolus (IRIFAFU) in patients with metastatic colorectal carcinoma: A Southern Italy Cooperative. Oncology Group phase III trial. Ann Oncol 16: 878-886, 2005.

28 Kasamatsu Y, Nakayama I and Kobayashi T: A case of lung cancer with hereditary angioedema treated effectively by chemoradiotherapy with $\mathrm{C} 1$ esterase inhibitor concentrate and danazol. Nihon Kokyuki Gakkai Zasshi 42(5): 435-439, 2004.

29 Christie DRH, Kirk JA, Clarke CL and Boyages J: Association of hereditary angioedema and hereditary breast cancer. Cancer Genet Cytogenet 95(2): 159-162, 1997.

30 Cugno M, Zanichelli A, Bellatorre AG, Griffini S and Cicardi M: Plasma biomarkers of acute attacks in patients with angioedema due to C1-inhibitor deficiency. Allergy 64(2): 254$257,2009$.

31 van Geffen M, Cugno M, Lap P, Loof A, Cicardi M and van Heerde W: Alterations of coagulation and fibrinolysis in patients with angioedema due to C1-inhibitor deficiency. Clin Exp Immunol 167(3): 472-478, 2012.

32 Csuka D, Veszeli N, Imreh É, Zotter Z, Skopál J, Prohászka Z, Varga L and Farkas H: Comprehensive study into the activation of the plasma enzyme systems during attacks of hereditary angioedema due to C1-inhibitor deficiency. Orphanet J Rare Dis 10: $132,2015$.
Received October 1, 2018

Revised October 19, 2018

Accepted October 26, 2018 